\title{
La efectividad de las páginas web en la comunicación empresarial de las pequeñas y medianas empresas. Un estudio en PYMES de la provincia de Cádiz
}

\author{
Webguneen eraginkortasuna enpresa txiki \\ eta ertainen enpresa-komunikazioan. Cádizko \\ probintziako zenbait ETEtan egindako ikerketa
}

\section{The effectiveness of websites in business communication of small and medium enterprises. A study in SMEs in the province of Cadiz}

Pedro Pablo Marín Dueñas ${ }^{1}$

María del Carmen Lasso de la Vega González ${ }^{2}$

zer

Vol. 22 - Núm. 42

ISSN: 1137-1102

e-ISSN: $1989-631 \mathrm{X}$

DOI: $10.1387 /$ zer. 17797 .

pp. 53-71

2017

Recibido el 31 de mayo de 2016, aceptado el 28 de marzo de 2017.

\section{Resumen}

Dada la importancia de la web para el desarrollo empresarial, esta investigación tiene como objetivo principal analizar los sites corporativos de una muestra de pequeñas y medianas empresas de la provincia de Cádiz Desde la perspectiva de la interactividad, la navegabilidad y los contenidos de las webs este trabajo medirá la eficacia que las webs tienen para facilitar la comunicación entre las pymes y sus públicos, a partir de la metodología mixta del análisis de contenido.

Palabras clave: Comunicación, web, interactividad, pymes, internet.

\section{Laburpena}

Kontuan izanda zer garrantzi duten webguneek enpresen garapenerako, ikerketa honen helburu nagusia da Cádizko probintziako enpresa txiki eta ertainen langin baten webgune

Universidad de Cádiz, pablo.marin@uca.es

2 Universidad de Cádiz, carmen.lasso@uca.es 
korporatiboak aztertzea. Elkarreraginaren, nabigagarritasunaren eta webguneen edukien ikuspegitik, lan honek webguneek ETEen eta haien hartzaileen arteko komunikazioa errazteko duten eraginkortasuna neurtuko du, eta, horretarako, edukien azterketaren metodologia mistoan oinarrituko da.

Gako-hitzak: Komunikazioa, web, interaktibitatea, ETEak, internet.

\begin{abstract}
Given the importance that the web is becoming for developing the communication of SMEs, this research has as main objective to analyze the corporate sites of a sample of small and medium enterprises of the province of Cadiz. From the perspective of interactivity, navigability and web content, this work will measure the effectiveness of it to facilitate communication between SMEs and their audiences, applying, for this, the mixed methodology of content analysis.
\end{abstract}

Keywords: Communication, web, interactivity, small and medium enterprises, internet. 


\section{Introducción}

En esta época de convulsiones y cambios, las Nuevas Tecnologías de la Información y la Comunicación (NTICS) han tenido un enorme impacto en los modelos de gestión empresarial. Internet, concretamente, ha modificado el tradicional paradigma comunicativo emisor-canal-receptor para darle protagonismo al usuario, a través de la interactividad que posibilita. Internet, con la web como máximo exponente, ha transformado las formas de comunicación entre las empresas y sus públicos, proporcionando beneficios para todos los sujetos implicados en el proceso comunicativo. Las empresas desarrollan conversaciones multidireccionales con sus usuarios que se convierten en protagonistas de la comunicación y en creadores de contenidos.

En este contexto, los sites empresariales se están consolidando como un fenómeno comunicativo cuyo impacto en las organizaciones está propiciando nuevas formas de establecer relaciones con sus públicos e, incluso, nuevas maneras de hacer negocio para las empresas. La web ofrece un nuevo medio de comunicación a las empresas para poder alcanzar sus objetivos y, dada la importancia que está alcanzando, es imprescindible su aplicación por parte de las compañías debido, entre otras, a la necesidad que tienen de comunicarse con sus públicos objetivos de forma eficaz. Se hace necesaria la implantación de páginas eficaces que favorezcan estas relaciones.

Ante la relevancia que este medio está adquiriendo para la comunicación de las empresas, se ha fijado como objeto de esta investigación el análisis de las páginas webs de las PYMES a la hora de facilitar las relaciones de las empresas con sus públicos.

La web se ha convertido en uno de los medios de comunicación con mayor atención por parte de las empresas, que se lanzan a la conquista de la misma no sólo como un medio de generación de ingresos, sino como una fuente para la difusión de la información y la comunicación (Alamandri y Mason, 2006).

$\mathrm{El}$ site corporativo de una empresa pasa a ser una herramienta imprescindible para todas las personas que se relacionan con ella configurándose, a su vez, como un instrumento básico para las organizaciones, proporcionando, entre otras, las siguientes oportunidades y beneficios (Castelló, 2010; García y Castillo, 2010; Ros, 2008; Díaz et al., 2008; Wang y Fesenmaier, 2006; Xing y Grandt, 2006; Yoon et al., 2006; Taylor y England, 2006; Schoenbach y Lauf, 2005; Harridge-March, 2004; Avlonitis y Karayanni, 2003; Berthon et al., 2003; Kozinets, 2002; Nuñez, 2001; Esrock y Leichty, 1999): Permite alcanzar ventajas competitivas; facilita realizar transacciones en tiempo real; hace que el negocio sea más visible y competitivo; influye en la imagen y credibilidad del negocio; permite la comunicación bidireccional, posibilidad de hablar directamente con los públicos; facilita el acceso al público objetivo; mejora la competitividad organizacional; reduce costes: tanto para la organización en sus procesos de producción como para los públicos en sus procesos de búsqueda de información; elimina los intermediarios; elimina las restricciones geográficas: las empresas están a un solo click, permiten dar a conocer a la organización a públicos que pueden estar en sitios muy lejanos; permite aumentar el número de clientes; hace más eficaz la comunicación, permitiendo desarrollar y construir relaciones más directas, personalizadas y a largo plazo; promociona los productos y servicios facilitando la consecución de los objetivos de marketing; desarrolla las Relaciones 
Públicas; permite dirigirse a cada consumidor con un mensaje personalizado; facilita la comparación con las webs de una amplia variedad de organizaciones; es el lugar más económico para publicitarse; permite participar en redes sociales; actualización a diario de la información: se pueden añadir productos, cambiar textos, incluir fotografías o eliminar cualquier aspecto; alta capacidad de afinidad; alto poder de microsegmentación; mediciones postcampaña.

El efecto que los sites corporativos ha causado en el mundo de la comunicación empresarial está fuera de toda duda y ha cambiado las estrategias comunicativas de muchas de ellas. Y otras, que antes no contaban con esta herramienta, empiezan a preocuparse por las mismas. Como apunta Castelló (2010), internet se configura como el medio idóneo para personalizar el mensaje y facilitar la interacción entre las empresas y sus públicos, cumpliendo las expectativas de éstos.

La necesidad de diferenciar la empresa hace necesaria una estrategia de imagen y comunicación online que ponga de manifiesto los esfuerzos de las empresas por integrar a sus públicos en la misma. Las compañías necesitan tener páginas que sean eficaces a la hora de permitir la comunicación con esos públicos. En este sentido, se puede afirmar que las variables que favorecen esa eficacia comunicativa entre la empresa y los usuarios son, fundamentalmente la usabilidad-navegabilidad, la interactividad y el contenido (Kaplanidou y Vogt, 2006; Ha y Love, 2005; Kline et al., 2004; Morrison et al., 2004; Yuan et al., 2004). Variables que tendrán un papel más que destacado puesto que, cualquier estrategia de comunicación basada en la web no podrá tener éxito si no se considera que es usable-navegable, interactiva y cuente con un contenido apropiado y útil para el usuario/lector.

El concepto de usabilidad -que incluye la accesibilidad o navegabilidad de la página- está directamente relacionado con la satisfacción del usuario (Hassan, 2006), ya que el diseño de un site puede convertirse en un primer obstáculo entre el usuario -receptor del mensaje- y la fuente de información. Por tanto, la usabilidad se configura como un elemento básico para la comunicación eficaz en este entorno. En sentido contrario, problemas en la usabilidad d pueden provocar que el usuario de por terminada la navegación (Sutcliffe, 2002).

Krug (2006) ofrece una definición sobre el término usabilidad según la cual no es más que estar seguro de que algo funciona bien. Es decir, que una persona con habilidades medias pueda utilizar una cosa con un fin concreto sin terminar frustrado. Entendida, en definitiva, como el grado de facilidad en el uso de los distintos elementos de un documento web, puede ser definida a partir de 5 atributos (Nielsen, 1993):

- Facilidad de aprendizaje. El usuario debe ser capaz de empezar a utilizarlo inmediatamente.

- Eficiencia. El usuario obtendrá un nivel de productividad alto al hacer un correcto uso.

- Retención sobre el tiempo. El uso del sitio, no debe olvidarse pasado un tiempo desde su aprendizaje.

- Tasas de error por parte de los usuarios. Minimizar los errores e indicar al usuario cómo solucionarlos en el caso de que se produzcan.

- Satisfacción subjetiva. ¿Les gusta el uso del sistema a los usuarios? 
Hassan (2006) considera que debe ser un factor transversal en el diseño de una web y que debe orientarse al usuario y a su no frustración, dotando a la misma de funcionalidad y proporcionando, además, un factor motivador. La usabilidad debe ser un factor determinante en la interacción del usuario ( $\mathrm{Li}$, et al. 2006) posibilitando que la empresa se presente correctamente ante sus públicos (Pollach, 2005).

Asociado al concepto de usabilidad se encuentra el de navegabilidad, definido como la facilidad para ir a un sitio y acceder a un área específica de la web (McKinney et al. 2002). Algunos factores que influyen de forma positiva en la navegación son la secuencia de las páginas, un diseño bien organizado y el consistente uso de los protocolos de navegación (Palmer, 2002). Como importante elemento del diseño, es una propiedad que refleja la capacidad de un site para ayudar al usuario a encontrar la información requerida (Huizingh, 2000; Marisco y Levialdi, 2004). Este factor, vinculado con la usabilidad que un sitio debe ofrecer a sus usuarios, abarca cuatro objetivos principales (Hernández, Martínez y De Hoyos, 2008): organizar y clasificar contenido, etiquetar información, diseñar sistemas de navegación y ayudar a los usuarios a encontrar información.

En definitiva, son numerosos los estudios precedentes que avalan la idea de la necesidad de integrar la usabilidad como un aspecto más de la correcta comunicación con los públicos de las empresas, para poder transmitir, así, una imagen fuerte y sólida que permita a los usuarios acceder y manejar fácilmente los mensajes que las empresas trasmiten a través de sus páginas corporativas (García y Castillo, 2010).

El segundo elemento básico para la comunicación eficaz de una web es la interactividad, considerada, además, como una de las características más importantes de Internet, así como uno de los pilares fundamentales de la comunicación 2.0. Requiere de la predisposición de la empresa a ayudar a sus públicos y el intercambio continuo de información entre ambas partes (retroalimentación), a través de los diferentes mecanismos que la empresa pone a disposición del usuario en el sitio -tales como teléfono, dirección postal, e-mail...-(Chen y Yen, 2004). Por lo tanto, la interactividad se configura como un elemento básico, determinando la eficacia de la página, y facilitando a la organización y al usuario el acceso y suministro de la información (Díaz et al., 2008).

Potenciar que el usuario quiera participar e interactuar con la web de la empresa debe convertirse en un objetivo a considerar por las organizaciones empresariales a la hora de desarrollar sus comunicaciones online ya que, como apuntan Huertas y Xifra (2009: 261), la interactividad puede desempeñar un rol crucial en internet fomentando y fortaleciendo las relaciones mutuas y la colaboración entre el emisor (la empresa) y el receptor (sus públicos objetivo).

Al hablar de interactividad se distingue entre dos niveles. Por un lado, la interactividad-personas, que es la que se desarrolla entre humanos, utilizando un determinado medio. En este sentido, la interactividad se refiere a las conversaciones bidireccionales que se establecen entre los seres humanos mediando en dicho diálogo un ordenador (Schultz, 2000). Por otro lado, se habla de la interactividad-máquina, que es aquella que tienen lugar entre humanos y máquinas (Hoffman y Novak, 2000). De esta forma, la interactividad con la máquina se centra en los elementos que proporcionan interacción entre los usuarios y el sitio web. Éstos pueden proporcionar esta interactividad a través de habitaciones de chat, mensajes instantáneos, o bien por 
medio de canales de comunicación como pueden ser e-mails o boletines (Li et al., 2006; Otim y Grover, 2006).

El último de los elementos clave a tener en cuenta cuando se habla de las web es el contenido de las mismas, referido a la cantidad y variedad de información presentada a través del site (Palmer, 2002). De forma más específica, se podría definir como la extensión, la minuciosidad, la precisión y la actualización de la información (Argawal y Venkatesh, 2002).

\section{Objetivos y metodología}

Partiendo de la premisa de que, como apuntan Gullikson et al. (1999), un diseño deficiente de una página web proyecta una imagen pobre de la organización al exterior afectándola de manera negativa, esta investigación pretende medir la gestión comunicativa de las PYMES en el entorno online a partir del diseño que hacen de sus portales corporativos en la red.

Más específicamente se quiere conocer la eficacia que las sedes online de las pequeñas y medianas empresas de la provincia de Cádiz tienen desde el punto de vista de la interactividad, la usabilidad/navegabilidad y los contenidos que ofrecen a la hora de facilitar las relaciones con los receptores de la comunicación, pues "la importancia del diseño se basa en que éste será el que modele la interacción entre usuario y aplicación" (Hassan et al., 2004).

Esta eficacia puede ser entendida, por tanto, como las posibilidades que a través de su concepción, diseño e implementación, ofrecen las webs como herramientas de comunicación que las PYMES utilizan en las relaciones con sus públicos,

Tal y como afirman Piñeiro-Naval, Igartúa y Marañón (2017) puede considerarse que existen dos maneras principales a la hora de realizar un análisis web. La evaluación heurística, en la que uno o varios especialistas chequean un site aplicándole un listado de criterios o ficha de análisis previamente definida; y las pruebas de rendimiento en las que se experimenta con una muestra de usuarios reales a los que se les puede aplicar también un cuestionario sobre su experiencia con la web.

En el caso de la presente investigación, se ha optado por un método basado en la evaluación heurística. Dejando de lado a los usuarios, se ha procedido a realizar un análisis de contenido de los sitios web. Se ha elegido este instrumento metodológico como técnica para estudiar las sedes corporativas de una muestra de PYMES gaditanas, por tratarse de una herramienta centrada en la identificación, codificación y categorización de información, de forma que el investigador sea capaz de conocer el objeto de estudio de forma objetiva y sistemática (García, 2012). Así mismo, permite "analizar fragmentos de texto segmentado en unidades que posteriormente serán codificadas” (García, Castillo y Carrillo, 2012: 163). Castillo (2007) refuerza la validez del análisis de contenidos en el estudio de sedes web aportando dos ventajas principales: permite acceder directamente a la fuente de información primaria, y además permite tratar el importante volumen de información que éstas contienen.

Siguiendo los pasos definidos por Piñuel (2002) a la hora de ejecutar un análisis de contenido, en primer lugar, el objeto de comunicación estudiado es la eficacia comunicativa de las páginas web. En segundo lugar, las tres categorías utilizadas, o grandes bloques conceptuales, son las que marcan la eficacia de una página web, 
como son: la usabilidad-navegabilidad, la interactividad y el contenido. En tercer lugar, se han definido las distintas unidades de análisis o indicadores, que permiten medir cada una de las categorías o dimensiones que han sido fijadas, y que ilustran las posibilidades comunicativas que ofrecen dichas webs para las empresas.

Para la selección de estos ítems se han tomando como referencia escalas de medida ya utilizadas y validadas por múltiples autores en investigaciones de relevancia, que han sido adaptadas al contexto específico de la presente investigación, y a partir de las cuales se ha diseñado una plantilla de análisis estructurada en tres bloques, uno para cada una de las categorías de análisis, incluyendo los indicadores para medir el contenido, la usabilidad-navegabilidad y la interactividad de la página web (tablas 1 y 2).

Tabla 1. Fuentes secundarias utilizadas para la creación de la plantilla de análisis.

\begin{tabular}{|c|c|c|}
\hline $\begin{array}{l}\text { CATEGORÍA } \\
\text { DE ANÁLISIS }\end{array}$ & NÚMERO DE INDICADORES & FUENTE BIBLIOGRÁFICA \\
\hline Contenido & 15 ítems & $\begin{array}{l}\text { Argawal y Venkatesh (2002) } \\
\text { Palmer (2002) }\end{array}$ \\
\hline Usabilidad & 25 ítems & $\begin{array}{l}\text { Nielsen y Tahir (2002) } \\
\text { Codina (2004) } \\
\text { Juliá, García y Polo (2004) } \\
\text { Moreno y Capriotti (2006) } \\
\text { Hernández, Martínez y De Hoyos } \\
\text { (2008) } \\
\text { Díaz, Martín y Esteban (2008) } \\
\text { Huertas y Xifra (2009) }\end{array}$ \\
\hline Interactividad & 21 ítems & $\begin{array}{l}\text { Castillo, Carrillo y Luna (2012) } \\
\text { García, Castillo y Carrillo (2012) } \\
\text { Gómez, Tapia y Díaz (2012) }\end{array}$ \\
\hline
\end{tabular}

Fuente: Elaboración propia.

El último de los pasos, siguiendo las recomendaciones de Piñuel (2002), a la hora de desarrollar el análisis de contenido, es la elección del sistema de recuento o medida. En el caso concreto de esta investigación se ha optado por seguir un sistema de valoración basado en los trabajos de Carrillo, Castillo y Luna (2012), por un lado, y de Nielsen y Tahir (2002), por otro. Así, el sistema de puntuación empleado ha sido el que se explica a continuación.

En primer lugar, Se ha valorado cada uno de los indicadores de acuerdo a la siguiente puntuación general:

0. Mala gestión o inexistencia del indicador

1. El indicador se aprecia de forma modesta

2. El indicador se aprecia de forma más notable

3. Buena gestión o existencia del indicador 
Tabla 2. Definición de indicadores y su relación con las categorías de análisis.

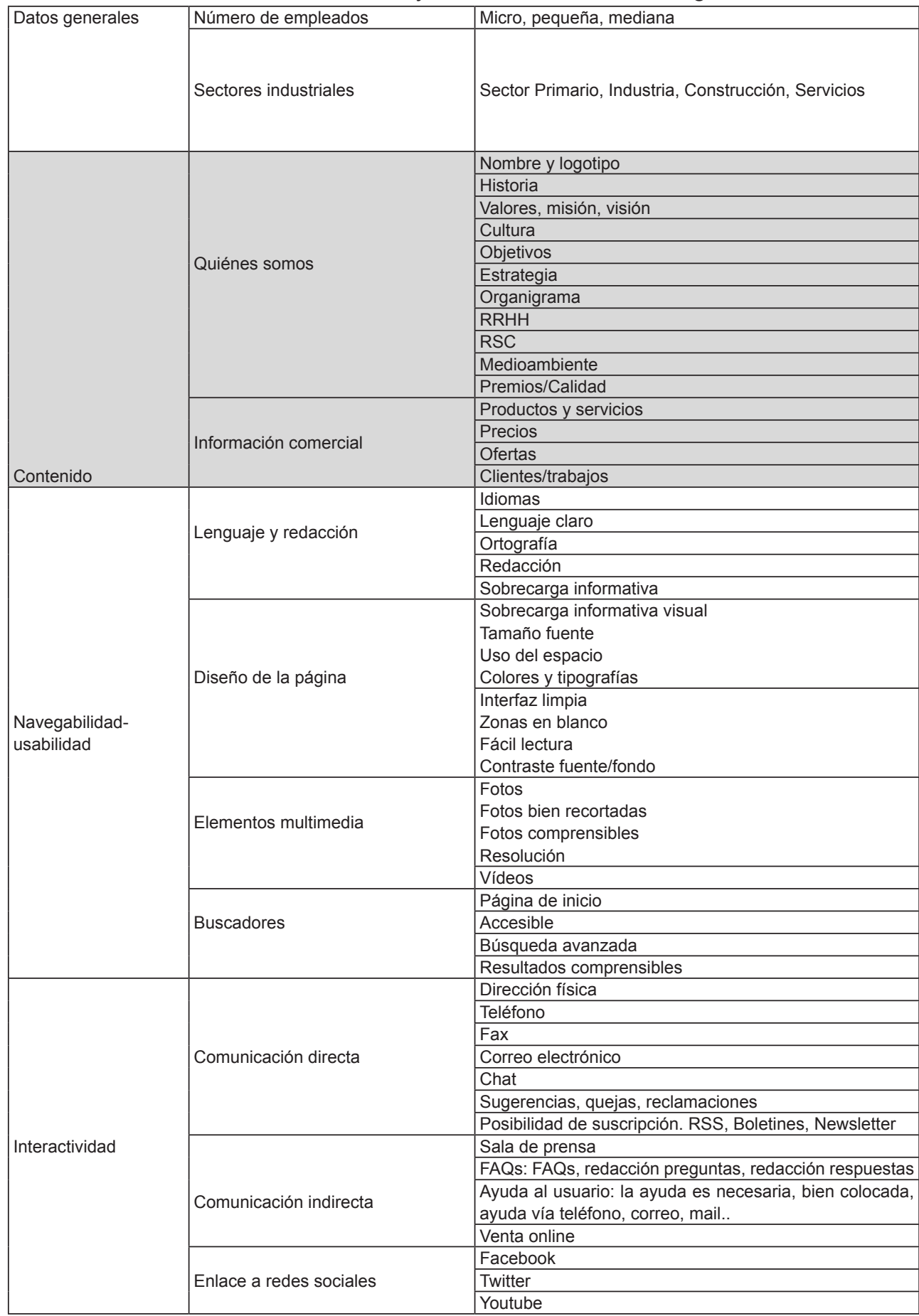


Una vez que han sido puntuados cada uno de esos ítems, y con el propósito de establecer clasificaciones de las webs de las empresas en función de su eficacia con una perspectiva de diseño, se ha determinado un baremo de medida. Para la elaboración de este baremo, en primer lugar, se han sumado los valores obtenidos para cada uno de los 61 ítems analizados (la máxima puntuación posible sería de 183 puntos) y el resultado ha sido dividido por el número total de indicadores que se han aplicado (en este caso 61). Para la obtención de un porcentaje, dicho resultado se ha multiplicado por 100 .

Finalmente, en función de esa cifra, que mide la eficacia, y siguiendo la propuesta realizada por Díaz, Martín y Esteban (2008), aunque añadiendo un intervalo más ${ }^{3}$, se ha considerado que:

- Una web que no alcance una puntuación de 45,75 (por debajo del 25\% de la máxima puntuación) sería una página empresarial con una muy baja eficacia a la hora de comunicarse con sus públicos.

- $\quad$ Una web con una puntuación entre 45,75 y 91,5 puntos (entre el 25\% y el $50 \%$ de la puntuación total) tendría una eficacia baja.

- $\quad$ Aquellas con una puntuación entre 91,5 y 137,25 puntos (entre el 50\% y el $75 \%$ ) son consideradas medianamente eficaces.

- Un site corporativo tiene una eficacia alta cuando alcanza una puntuación superior a 137,25 puntos (75\% o más del total de la puntuación).

Tabla 3. Número de ítems de medida y puntuación.

\begin{tabular}{|l|l|l|}
\hline CATEGORÍA DE ANÁLISIS & NÚMERO DE ÍTEMS & PUNTUACIÓN MÁXIMA \\
\hline Contenido & 15 ítems & 45 puntos \\
\hline Usabilidad/Navegabilidad & 25 ítems & 75 puntos \\
\hline Interactividad & 21 ítems & 63 puntos \\
\hline
\end{tabular}

Fuente: Elaboración propia.

Tabla 4. Baremos para la medición de la eficacia.

\begin{tabular}{|l|l|l|}
\hline NIVEL DE EFICACIA & INTERVALOS & $\%$ SOBRE EL TOTAL \\
\hline Eficacia alta & $137,23-183$ puntos & $75 \%-100 \%$ \\
\hline Eficacia media & $91,5-137,23$ puntos & $50 \%-75 \%$ \\
\hline Eficacia baja & $45,75-91,5$ puntos & $25 \%-50 \%$ \\
\hline Eficacia muy baja & $0-45,75$ puntos & $0 \%-25 \%$ \\
\hline
\end{tabular}

Fuente: Elaboración propia.

A modo de ejemplo, una página corporativa que haya obtenido una puntuación total de 100 puntos como resultado de la suma de las valoraciones de cada uno de los ítems, tendría una puntuación porcentual final de 54,64\% sobre el máximo de puntos posibles. Por lo que se consideraría que la eficacia que tiene para las PYMES a la hora de comunicarse con sus públicos sería media.

\footnotetext{
3 Se ha añadido el intervalo "eficacia muy baja" con respecto a la propuesta de Díaz et al. (2008).
} 
Una vez definidos cada uno de los principales aspectos metodológicos para realizar un correcto análisis de contenido, se procedió a la aplicación del mismo sobre una muestra previamente definida. Para la determinación de la misma y considerando que se trata de un estudio de carácter exploratorio, se seleccionó una muestra no representativa conformada por pequeñas y medianas empresas ubicadas en la provincia de Cádiz según la información proporcionada por la base de datos $\mathrm{SABI}^{4}$ que en la fecha del estudio incluía 2.373 empresas con sede web activa en dicha región y de la que se seleccionaron 50 PYMES de manera aleatoria, siendo conscientes de que "internet convierte en una tarea harto complicada el diseño de una muestra que represente el universo del que procede" (Piñeiro-Naval et al., 2017).

La composición final de la muestra, atendiendo al número de empleados y al sector en el que desarrollan su actividad, se define de la siguiente manera:

En cuanto al número de empleados, las empresas elegidas son 29 Micro empresas (0-9 empleados) (58\%), 15 Pequeñas empresas (10-49 empleados) (30\%) y 6 Medianas empresas (50-249 empleados) (12\%). Respecto al sector industrial en el que desarrollan su actividad, 6 de ellas pertenecen al Sector Primario (12\%), 12 a la Industria (24\%), 2 a la Construcción (4\%) y 30 al Sector Servicios (60\%).

Por último se llevó a cabo la tabulación y depuración de los datos así como el análisis estadístico de los mismos. Para el análisis de los datos y de cara al tratamiento estadístico de los mismos se ha utilizado la herramienta estadística SPSS.

\section{Resultados}

Para ofrecer una perspectiva más completa de los datos derivados del análisis de contenido, se va a proceder, en primer término, al estudio individualizado de cada una de las tres categorías, de cara a conocer la eficacia para cada una de ellas para, posteriormente, presentar un único índice que determine la eficacia de las webs de las pequeñas y medianas empresas de la provincia de Cádiz.

En términos generales, los sites corporativos, desde el punto de vista del contenido, son muy mejorables, siendo un $64 \%$ el número de páginas con una eficacia baja-muy baja. El número de empresas que cuentan con una web con una eficacia alta es tan sólo de 4 (8\%).

Las empresas se identifican de manera correcta en sus portales. El 94\% muestran el nombre y/o el logotipo de la PYME La identidad visual de las empresas queda clara a través de su logo, ubicado, además, en un lugar identificable y visible. Es notable el número de PYMES que ofrecen información sobre su historia. Ya sea de manera breve o con una mayor descripción, el 94\% de las webs contienen algún contenido referido a este aspecto. Dentro de la información ofrecida, en general, la historia ocupa un lugar importante y se suele hacer hincapié en los orígenes (con especial mención de los fundadores) así como los hechos más importantes que han acontecido en la organización.

Otros parámetros, referidos sobre todo a los aspectos más corporativos y relacionados con la identidad de la organización y su desarrollo estratégico tales como los valores, la misión y la visión o la cultura corporativa están poco presentes en las webs de las pequeñas y medianas empresas. El indicador relativo a medir la

${ }^{4}$ https://sabi.bvdinfo.com/version-2015211/home.serv?product=sabineo 
presencia de información sobre el organigrama ofrece unos resultados un tanto mejores que los anteriores, aunque no se puede afirmar que esté bien gestionado. El 58\% de las Webs ofrecen algo de información personal relativa a su organigrama, motivado, posiblemente, por el hecho de querer dotar de cierto carácter de cercanía con el público al mostrar a las personas que forman parte de la empresa.

Los indicadores relacionados con los productos/servicios que ofrecen las empresas están mucho más presentes en las webs de las pequeñas y medianas empresas. En el $72 \%$ de los casos analizados se aprecian de forma, como mínimo, notable. Si bien la información relativa a precios o promociones está peor gestionada, y no se usa la Web para mostrar a los usuarios posibles promociones que la empresa ofrezca, si que se aprecia una presencia de información relacionada con los clientes y trabajos que han realizado, a modo de muestra, y como prueba de fiabilidad de la PYME para los usuarios que visiten la web.

De los resultados derivados de la categoría usabilidad-navegabilidad se desprende que los portales corporativos tienen una usabilidad aceptable y poseen unos índices de navegabilidad adecuados (el 58\% de las mismas tienen una eficacia media-alta). Son páginas fáciles de navegar, de diseño sencillo y bien estructurado. Se aprecia, en general, una claridad en la presentación de la información, caracterizada por un buen contraste y una correcta legibilidad. Las webs están perfectamente identificadas a partir de la dirección URL coincidiendo en la mayoría de los casos totalmente con el nombre de la empresa lo que facilita enormemente su recuerdo y permitiendo transmitir una única marca y un único nombre. En un plano más técnico, ofrecen, en general, una adecuada compatibilidad con los distintos navegadores que ofrece el mercado. La gestión de los indicadores relacionados con el lenguaje y la redacción están bien tratados. Tanto la corrección ortográfica, como la claridad y la sencillez del lenguaje empleado ofrecen datos positivos. Lo mismo ocurre con las cuestiones de estilo y la gestión de la información que se ofrece. Las PYMES eligen bien el lenguaje utilizado para comunicarse con sus públicos facilitando la mejor transmisión de los mensajes, que llegan al usuario mediante un lenguaje familiar y cercano.

Los indicadores que se han definido para medir tanto la sobrecarga informativa visual como la amigabilidad de la interfaz consiguen ofrecer la información sin cargar demasiado al usuario y a través de una interfaz sin mucho ruido visual. El diseño de la página, desde el punto de vista de un usuario, puede considerarse correcto, lo que agiliza la lectura en pantalla, evitando que el usuario cierre la página nada más acceder a ella, evitando la fatiga en la búsqueda de información y la sensación de densidad informativa.

La mayoría de las webs $(80 \%)$ cuenta con fotografías, que ilustran los contenidos expuestos, mostrando imágenes de sus productos o servicios, y cuya función principal es la de acompañar a los contenidos textuales. En general, muestran elementos que tienen relación en el contexto (es decir que son fotos relativas a la empresa tales como la sede, los productos que ofrece, etc.) pero que, en general, no aportan un valor añadido real ya que realizan una función de meros acompañantes del texto. Por el contrario, no se le presta mucha atención a los vídeos, siendo su presencia muy baja (en tan sólo 9 de las webs analizadas se ha detectado información sobre la empresa en formato vídeo). 
La desagregación atendiendo a la usabilidad deja, sobre todo un resultado destacable: son 15 las webs empresariales que alcanzan una eficacia alta ${ }^{5}$ desde el punto de vista de la navegación de las mismas. Disminuyen, en consecuencia, el número con una eficacia media, baja y muy baja. En el caso de la eficacia muy baja, sólo 6 de las analizadas se sitúan en esta posición. Estos datos han de entenderse como positivos, ya que la usabilidad es considerada un aspecto clave en la creación de una buena imagen.

Las webs que se perciben como más fáciles de abrir, navegar o utilizar crean una actitud y una imagen más favorable entre los usuarios. Los usuarios que navegan con mayor facilidad y encuentran la información que desean rápidamente, obtienen una mejor impresión de la empresa y satisfacen en mayor grado sus expectativas (Huertas y Xifra, 2009). Finalmente, la distribución de los resultados atendiendo a la interactividad deja una conclusión muy clara: el nivel de eficacia de la interactividad es muy bajo (el 60\%). De hecho el 74\% de las páginas corporativas tienen una interactividad baja o muy baja.

La presencia de los mecanismos clásicos de comunicación con la empresa tales como la dirección, el teléfono y el email están presentes. Las pequeñas y medianas empresas son conscientes de la necesidad de facilitar a sus públicos la forma de ponerse en contacto con ellas, ya sea presencialmente o a través de una llamada o un correo. Por el contrario, y debido a la evolución de las Nuevas Tecnologías de la Información y la Comunicación -sobre todo el correo electrónico-, el fax está perdiendo importancia, aunque aún está presente en casi la mitad de las PYMES. Otros mecanismos, más en línea con la web 2.0, tales como las RSS, los boletines o las Newsletter, por no hablar de los chats interactivos, apenas son considerados y su presencia es prácticamente residual. Tampoco ofrecen mecanismos específicos para gestionar sugerencias, quejas o reclamaciones, aunque en comparación con las otras herramientas, tiene una mayor presencia.

La presencia de los indicadores que miden la comunicación indirecta a través del portal corporativo es también baja. La existencia de salas de prensa, de una sección destinada a preguntas frecuentes o de ayuda al usuario es baja. Otra de las ventajas que ofrece la web para las empresas es la posibilidad de vender sus productos y servicios directamente, sin necesidad del contacto directo con el usuario. Pero son pocas (tan sólo el $26 \%$ de la muestra) las que habilita en sus sedes esta posibilidad. Tan sólo en 13 webs de las 50 analizadas se puede encontrar una sección para la venta online y para la realización de pedidos y reservas en línea.

El último de los indicadores estudiados desde el punto de vista de la interactividad es la presencia o no de un enlace a alguna de las redes sociales más comunes, tales como Facebook, Twitter y Youtube. La primera de ellas está presente de una forma notable (el 62\% habilitan un enlace a Facebook). Por el contrario, en el caso de Twitter, sólo el $38 \%$ cuenta con dicho enlace.

\footnotetext{
Se han incluido las Webs con una puntuación de 74,7, por su proximidad al umbral del $75 \%$.
} 
Tabla 5. Eficacia web en la pequeña y mediana empresa.

\begin{tabular}{|c|c|c|c|c|c|}
\hline \multicolumn{5}{|c|}{ ÍNDICE GLOBAL DE LA EFICACIA DE LAS WEBS EMPRESARIALES } & \multirow[b]{2}{*}{$\%$} \\
\hline & CONTENIDO & USABILIDAD / NAVEGABILIDAD & INTERACTIVIDAD & PUNTUACIÓN & \\
\hline WEB 1 & 18 & 56 & 21 & 95 & 51,9 \\
\hline WEB 2 & 20 & 31 & 15 & 66 & 36,1 \\
\hline WEB 3 & 8 & 14 & 6 & 28 & 15,3 \\
\hline WEB 4 & 3 & 39 & 12 & 54 & 29,5 \\
\hline WEB 5 & 30 & 59 & 31 & 120 & 65,6 \\
\hline WEB 6 & 10 & 14 & 9 & 33 & 18,1 \\
\hline WEB 7 & 26 & 56 & 38 & 120 & 65,6 \\
\hline WEB 8 & 9 & 38 & 6 & 53 & 28,9 \\
\hline WEB 9 & 21 & 51 & 48 & 120 & 65,6 \\
\hline WEB 10 & 38 & 60 & 34 & 132 & 72,1 \\
\hline WEB 11 & 10 & 17 & 9 & 36 & 19,7 \\
\hline WEB 12 & 7 & 38 & 6 & 51 & 27,9 \\
\hline WEB 13 & 13 & 27 & 9 & 49 & 26,7 \\
\hline WEB 14 & 26 & 59 & 39 & 124 & 67,7 \\
\hline WEB 15 & 18 & 52 & 15 & 85 & 46,4 \\
\hline WEB 16 & 11 & 21 & 9 & 41 & 22,4 \\
\hline WEB 17 & 1 & 44 & 9 & 54 & 29,5 \\
\hline WEB 18 & 26 & 54 & 31 & 111 & 60,6 \\
\hline WEB 19 & 9 & 9 & 12 & 30 & 16,4 \\
\hline WEB 20 & 24 & 56 & 34 & 114 & 62,3 \\
\hline WEB 21 & 9 & 36 & 3 & 48 & 26,3 \\
\hline WEB 22 & 28 & 57 & 33 & 118 & 64,5 \\
\hline WEB 23 & 10 & 26 & 9 & 45 & 24,6 \\
\hline WEB 24 & 5 & 38 & 9 & 52 & 28,4 \\
\hline WEB 25 & 8 & 27 & 12 & 47 & 25,7 \\
\hline WEB 26 & 34 & 57 & 26 & 117 & 63,9 \\
\hline WEB 27 & 20 & 56 & 12 & 88 & 48,1 \\
\hline WEB 28 & 12 & 20 & 9 & 41 & 22,4 \\
\hline WEB 29 & 23 & 63 & 26 & 112 & 61,2 \\
\hline WEB 30 & 22 & 29 & 12 & 63 & 34,4 \\
\hline WEB 31 & 10 & 24 & 15 & 49 & 26,8 \\
\hline WEB 32 & 33 & 63 & 33 & 112 & 61,2 \\
\hline WEB 33 & 21 & 61 & 12 & 94 & 51,3 \\
\hline WEB 34 & 23 & 54 & 20 & 97 & 53,1 \\
\hline WEB 35 & 13 & 25 & 12 & 50 & 27,3 \\
\hline WEB 36 & 1 & 47 & 9 & 57 & 31,1 \\
\hline WEB 37 & 37 & 55 & 33 & 125 & 68,3 \\
\hline WEB 38 & 10 & 9 & 9 & 28 & 15,3 \\
\hline WEB 39 & 32 & 54 & 40 & 126 & 68,8 \\
\hline WEB 40 & 7 & 34 & 9 & 50 & 27,3 \\
\hline WEB 41 & 29 & 57 & 50 & 136 & 74,3 \\
\hline WEB 42 & 10 & 18 & 9 & 37 & 20,2 \\
\hline WEB 43 & 11 & 33 & 6 & 50 & 27,3 \\
\hline WEB 44 & 10 & 21 & 9 & 40 & 21,8 \\
\hline WEB 45 & 31 & 57 & 31 & 119 & 65,1 \\
\hline WEB 46 & 38 & 49 & 36 & 123 & 67,2 \\
\hline WEB 47 & 12 & 19 & 12 & 43 & 23,4 \\
\hline WEB 48 & 28 & 53 & 44 & 125 & 68,3 \\
\hline WEB 49 & 5 & 31 & 3 & 39 & 21,3 \\
\hline WEB 50 & 30 & 57 & 43 & 130 & 71,1 \\
\hline
\end{tabular}

Fuente: Elaboración propia. 
Tabla 6. Distribución de la eficacia Web en la pequeña y mediana empresa.

\begin{tabular}{|l|l|l|l|l|}
\hline \multicolumn{2}{|l|}{ DISTRIBUCIÓN POR NIVEL DE EFICACIA, TAMAÑ Y SECTOR DE ACTIVIDAD } \\
\hline NIVEL DE EFICACIA & N $^{\circ}$ DE WEBS & $\%$ & $\begin{array}{l}\text { TAMAÑO } \\
\text { DE LAS } \\
\text { EMPRESAS }\end{array}$ & $\begin{array}{l}\text { SECTOR } \\
\text { DE ACTIVIDAD }\end{array}$ \\
\hline Eficacia alta & 1 & $2 \%$ & $\begin{array}{l}\text { Micro: } 1 \\
\text { Pequeña:0 } \\
\text { Mediana: 0 }\end{array}$ & $\begin{array}{l}\text { Sector Primario: 0 } \\
\text { Industria: 0 } \\
\text { Construcción: 0 } \\
\text { Servicios: 1 }\end{array}$ \\
\hline Eficacia media & 20 & $40 \%$ & $\begin{array}{l}\text { Micro: 10 } \\
\text { Pequeña:8 } \\
\text { Mediana: 2 }\end{array}$ & $\begin{array}{l}\text { Sector Primario: 3 } \\
\text { Industria: 6 } \\
\text { Construcción: 0 } \\
\text { Servicios: 11 }\end{array}$ \\
\hline Eficacia baja & 18 & $36 \%$ & $\begin{array}{l}\text { Micro: 9 } \\
\text { Pequeña:5 } \\
\text { Mediana:4 }\end{array}$ & $\begin{array}{l}\text { Sector Primario: 1 } \\
\text { Industria: 4 } \\
\text { Construcción: 0 } \\
\text { Servicios: 13 }\end{array}$ \\
\hline Eficacia muy baja & 11 & $22 \%$ & $\begin{array}{l}\text { Micro: 9 } \\
\text { Pequeña:2 } \\
\text { Mediana:0 }\end{array}$ & $\begin{array}{l}\text { Industria: 2 } \\
\text { Construcción: 2 } \\
\text { Servicios: 5 }\end{array}$ \\
\hline
\end{tabular}

Fuente: Elaboración propia.

Según esta clasificación general que se ha presentado, la web más eficaz ha alcanzado una puntuación de 136 puntos, lo que significa el 74,3\% de los puntos totales. Según el baremo utilizado, aunque no llegue al límite de $75 \%$ de la puntuación total se ha considerado que esta Web tiene una eficacia alta teniendo en cuenta la usabilidad, la interactividad y el contenido. Dicha Web se corresponde con una microempresa del sector servicios.

20 de las 50 analizadas (40\% de la muestra) alcanzan una puntuación superior a los 91,5 puntos mínimos necesarios para considerar su eficacia media. Del resto (18 en total) han conseguido una puntuación inferior a los 91,5 puntos (50\% de la puntuación total) pero superior a 45,75 puntos considerándose por tanto que estas Webs tienen una eficacia baja.

Finalmente, se considera que la eficacia de 11 portales corporativos es muy baja al no alcanzar el límite de 45,75 puntos (25\% de la puntuación máxima). En este sentido, dos obtienen la peor puntuación, alcanzando tan sólo 28 puntos (un 15,3\% del total).

En términos generales, podría considerarse que las webs de las pequeñas y medianas empresas tienen una eficacia media-baja. El $76 \%$ de la muestra está dentro de este intervalo. El aspecto positivo es que el $40 \%$ de las PYMES tienen un site corporativo con una eficacia media. El lado negativo es que el $58 \%$ de las estudiadas tienen una eficacia baja o muy baja.

\section{Conclusiones}

Al margen de los datos cuantitativos, en términos generales las pequeñas y medianas empresas deben potenciar la interactividad de sus portales corporativos, lo que 
contribuirá a la creación de relaciones a largo plazo. Las empresas deben olvidar su visión cortoplacista y aprovechar las oportunidades que ofrece el entorno 2.0 para fomentar vínculos duraderos con los públicos interesados en interactuar con ellos.

Deben, por otro lado, mejorar los contenidos que ofrecen. Las PYMES deben aprovechar la web para proyectar su imagen corporativa. Para ello deberían reservar una sección destinada a comunicar cual es la identidad de la organización, con información precisa que contribuya a configurar una imagen correcta. Como se ha dicho anteriormente, la identidad corporativa de una empresa debe ser el pilar sobre el que se sustente la información mostrada en el portal y las empresas tienen que ser conscientes de la necesidad de contar con una sección destinada a la presentación de dicha identidad.

Tratando de hacer un balance global se podría señalar que la mayoría de las empresas analizadas ofrecen información muy escueta sobre ella misma. Presentan información bastante limitada, sobre todo en lo referente a la identidad de la organización (objetivos, misión, visión, proyecto), centrándose, la mayoría, en ofrecer información de carácter comercial. En cambio, los datos reflejan la importancia que tiene para las pequeñas y medianas empresas contar con espacios donde ofrecer sus productos/servicios, siendo la web una gran plataforma para informar a los públicos sobre lo que las PYMES hacen. A tenor de los resultados, la Web se ha convertido, por tanto, en un medio adecuado para desarrollar la Comunicación Comercial de las pequeñas y medianas empresas.

Aunque la calidad de la información, en general, no es mala, si que es mejorable. Como aspecto positivo se puede entender la actualización de los contenidos. Las PYMES analizadas aportan información puntualmente actualizada que favorece las visitas de los usuarios.

La generalidad y la imprecisión de los contenidos mostrados en las webs empresariales es, sin duda, un aspecto a mejorar. Deben, en definitiva, destinar recursos, tanto tecnológicos como de personal a la comunicación a través del portal empresarial, desarrollando estrategias comunicativas online con el site corporativo como máximo escaparate.

La categoría interactividad es, si se comparan las tres analizadas, la que ofrece peores resultados. A pesar de ser una de las principales características de Internet, que permite fomentar y fortalecer las relaciones entre las empresas y sus públicos, en general, se puede afirmar, a tenor de los resultados, que las webs de las pequeñas y medianas empresas son poco interactivas, primándose la información. Se trata de una comunicación unidireccional si bien facilitan al usuario ponerse en contacto con la empresa a través del correo electrónico o el teléfono.

Las PYMES observadas sitúan su aproximación al usuario online en un escalón muy inicial. Proporcionan apenas dos o tres (teléfono, dirección postal, email) formas de contacto con la empresa, pero ni segmentan públicos ni permiten una interactividad entendida desde una perspectiva 2.0. Si bien algunas permiten la venta online, se pueden considerar, más como un catálogo de ventas trasladado del entorno offline a la red, en el que informan sobre sus productos y servicios que como un servicio especializado.

Desde un punto de vista positivo sí que se puede entender el hecho de que Internet se ha convertido en un medio muy utilizado por las pequeñas y medianas empre- 
sas para el desarrollo de su actividad comunicativa. Disponer de página web y el uso del correo electrónico, así como tener presencia en las redes sociales mayoritarias como Facebook o Twitter es una realidad en este tipo de empresas.

Si bien la web se convierte, fundamentalmente, en un espacio donde ofrecer los productos y servicios de la empresa, sí que utilizan este medio para trasladar, aunque sea mínimamente, la identidad de las empresas a todos los públicos. A través del site corporativo las pequeñas y medianas empresas ofrecen información de tipo corporativo (su historia fundamentalmente) y trasladan su imagen a los visitantes de estas páginas, a través de su identidad visual, aunque es cierto que otros aspectos relacionados con la misma están muy poco presentes.

Más allá de que la gestión de las páginas sea más o menos adecuada (del análisis de contenido se concluye que las webs de las pequeñas y medianas empresas presentan una eficacia baja) parece que, en este caso, no sólo son conscientes de la importancia del nuevo medio sino que, además, también lo utilizan, lo que supone un punto de partida muy importante puesto que, desde ahí, sólo queda mejorar.

\section{Referencias bibliográficas}

Alamandri, F. y Mason, K. (2006). The future of airline distribution. Journal of Air Transport Management, 12(3), 122-134.

Argawal, R. y Venkatesh, V. (2002). Assesing a firm's Web presence: a heuristic evaluation procedure for the measurement of usability. Information Systems Research, 13(2), 168-186.

Avlonitis, G. y Karayanni, D. (2003). The use of Internet in business to business marketing: some evidence from American and European companies. Atenas: University of economics and Business.

Berthon, P., Ewing, M., Pitt, L. y Naude, P. (2003). Understanding B2B and the Web: the acceleration of coordination and motivation. Industrial Marketing Management, 32(7), 553-561.

Carrillo, M. V., Castillo, A. y Luna, M. R. (2012). Estudio de las sedes webs de PYMES mexicanas como herramienta de apoyo a sus estrategias de comunicación empresarial. Comunicación estratégica y organizacional. Revisión de estrategias en comunicación organizacional. Recuperado de http://www.ae-ic.org/ tarragona2012/contents/comunicacions_cd/ok/63.pdf

Castelló, A. (2010). Estrategias empresariales en la web 2.0. Las redes sociales online. Alicante: Editorial Club Universitario.

Castillo, A. (2007). La comunicación de las marcas universitarias españolas a través de sus sedes webs. Tesis doctoral. Universidad de Extremadura.

Chen, K. y Yen, D.C. (2004). Improving the quality of online presence through interactivity. Information \& Management, 42, 217-226. 
Codina, L. (2004). Hiperdocumentos: composición, estructura y evaluación. En J. Díaz y R. Salaverría (coords.), Manual de Redacción Ciberperiodística, (pp. 141192). Barcelona: Ariel Comunicación.

Díaz, E., Martín, D. y Esteban, A. (2008). Evaluación de la eficacia de las páginas web: un análisis de contenido de las principales compañías aéreas. En Universidad, Sociedad y Mercados Globales. Recuperado de http://dialnet.unirioja.es/ servlet/articulo?codigo $=2751745$.

Esrock, S. y Leichti. (1999). Corporate World Wide Web pages: serving the new media and others publics. Journalism \& Mass Communication Quarterly, 76(3), 456-467.

García, M. y Castillo, A. (2010). Webs usables y accesibles en PYMES. Retos para el futuro. Revista Latina de Comunicación Social, 65, 392-409.

García, M., Castillo, A. y Carrillo, M. V. (2012). La interactividad en las sedes webs corporativas: retos y oportunidades para las pymes. Perspectivas em Ciência da Informação, 17(2), 160-174.

Gómez, B., Tapia, A. y Díaz, O. (2012). La Comunicación corporativa a través de las páginas Web: el caso de las ONGS españolas. Revista de Comunicación Vivat Academia. XIV (120), 1-20.

Harridge-March, S. (2004). Electronic marketing, the new kid on the block. Marketing Intelligence and Planning, 22(3), 279-309.

Hassan, Y., Martín, F. J. y Iazza, G. (2004). Diseño web centrado en el usuario: usabilidad y arquitectura de la información. Hipertext, 40. Recuperado de https:// www.upf.edu/hipertextnet/numero-2/diseno_web.html

Hassan, Y. (2006). Factores del diseño web orientado a la satisfacción y no frustración de uso. Revista Española de Documentación Científica, 24(1), 239-257.

Hernández, B., Martínez, J. y De Hoyos, J. (2008). Calidad de la información Web en la banca electrónica. International Congress Marketing Trends. Venice.

Hoffman, D.L. y Novak, T.P. (2000). Marketing in hypermedia computer mediated environments: conceptual foundations. Journal of Marketing, 60(3), 50-68.

Huertas, A. y Xifra, J. (2009). ¿Marcas o genéricos? La comunicación en línea de las marcas farmacéuticas. ZER Revista de Estudios de Comunicación, 14(27), 251-270.

Huizingh, E. (2000). The content design of web sites: an empirical study. Information \& Management, 37, 123-134.

Juliá, J. F., García, G. y Polo, F. (2004). La información divulgada a través de Internet por las cooperativas. CIRIEC-España, Revista de Economía Pública, Social y Cooperativa, 49, 167-192.

Kaplanidou, K. y Vogt, C. A. (2006). Structural Analysis of Destination Travel Intentions as a Function of Website features. Journal of Travel Research, 45(2), 204-216.

Kozinets, R. (2002). The field behind the screen: Using netnography for Marketing research in online communities. Journal of Marketing Research, 39(1), 61-72.

Krug, S. (2006). No me hagas pensar. Una aproximación a la usabilidad en la web. Madrid: Pearson Educación.

Li, D., Browne, G. y Chau, P. (2006). An empirical investigation of web site use using a commitment-based model. Decision Sciences, 37(3), 196-211. 
Marsico, M. y Levialdi, S. (2004). Evaluating web sites: exploiting user's expectations. Int. Journal of Human Computer Studies, 60, 381-416.

Mckinney, V., Yoon, K. y Zahedi, F. (2002). The measurement of Web-customer satisfaction: an expectation and disconfirmation approach. Information Systems Research, 13(3), 296-315.

Moreno, A. y Capriotti, P. (2005). La comunicación de las empresas españolas en sus webs corporativas. Análisis de la información de responsabilidad social, ciudadanía corporativa y desarrollo sostenible. ZER Revista de Estudios de Comunicación, 21, 47-62.

Nielsen, J. (1993). Usability engineering. Morgan Kaufmann.

Nielsen, J. y Tahir, M. (2002). Usabilidad de páginas de inicio: análisis de 50 sitios Web. Madrid: Pearson Educación.

Nuñez, L. (2001). La gestión de clientes en el comercio electrónico. Aplicación de Algoritmos Genéticos (AG) al CRM. Economía Industrial, 340, 83-92.

Otim, S. y Grover, V. (2006). An empyirical study on Web-based services and customer loyalty. European Journal of Information Systems, 15(6), 527-541.

Palmer, J. (2002). Web site usability, design and performance metrics. Information System Research, 13(2), 151-167.

Piñeiro-Naval, V., Igartua, J. J. y Marañón, F. (2017). El diseño de las sedes web municipales de España. Una propuesta metodológica para su análisis. Revista Española de Documentación Científica, 40(1).doi: http://dx.doi.org/10.3989/ redc.2017.1.1368

Piñuel, J.L. (2002). Epistemología, metodología y técnicas de análisis de contenido. Estudios de Sociolingüística, 3, 1-42.

Pollach, I. (2005). Corporate self-presentation on the WWW. Strategies for enhancing usability, credibility and utility. Corporate Communications: An international Review, 10(4), 285-301.

Ros, V. (2008). E-branding. Posiciona tu marca en la Red. Oleiros (A Coruña): Netbiblo.

Schoenbach, K. y Lauf, E. (2005). Another look at the trap effect of television and beyond. International Journal of Public Opinion Research, 16(2), $169-182$.

Schultz, T. (2000). Mass media and the concept of interactivity: an exploratory study of online forums and reader email. Media Culture \& Society, 22, 205-221.

Sutcliffe, A. (2002). Assessing the Reliability of Heuristic Evaluation for Website Attractiveness and Usability. En Proceedings of the 35th Hawaii International Conference on System Sciences, Hawaii.

Taylor, M. y England, D. (2006). Internet Marketing: website navigational design issues. Marketing Intelligence and Planning, 24(1), 77-85.

Wang, Y. y Fesenmaier, D. (2006). Identifying the success factors of web-based marketing strategy: an investigation of convention and visitors bureaus in the United States. Journal of Travel Research, 44(3), 239-249.

Xing, Y. y Grandt, D. (2006). Developing a framework for measuring physical distribution service quality of multi-channel and pure player internet retailers. International Journal of retail and Distribution Management, 34(4/5), 278-289. 
La efectividad de las páginas web en la comunicación empresarial de las pequeñas y medianas empresas. Un estudio en ...

Yoon, M., Young, D. y Won, T. (2006). Impact of e-business on air travel markets: Distributions of airline tickets in Korea. Journal of Air Transport Management, 12(5), 31-46. 